\title{
Vídeos como ferramentas de educação em saúde bucal em tempos de pandemia na integração ensino e serviço do Centro Universitário de Patos de Minas
}

\author{
Maria Teresa Borges Araújo*; Carolina Silva Pereira*; Cássia Eneida Souza Vieira Dutra**; Thays \\ Cristiny Simão Melo**; Ivânia Aparecida Pimenta**; Thiago de Amorim Carvalho** \\ * Estudante, Curso de Graduação em Odontologia, Centro \\ Universitário de Patos de Minas \\ ** Docente, Curso de Graduação em Odontologia, Centro \\ Universitário de Patos de Minas
}

Recebido: 29/06/2021. Aprovado: 08/11/2021.

\begin{abstract}
RESUMO
No curso de graduação em Odontologia do Centro Universitário de Patos de Minas, diversas atividades do estágio Integração Ensino, Serviço e Comunidade (INESC) tiveram que ser suspensas em sua forma presencial, conforme recomendações das autoridades sanitárias, devido à pandemia da COVID-19. O objetivo deste artigo é relatar a experiência da aplicação dos conceitos de educação em saúde no ensino remoto por meio da confecção de vídeos de criação compartilhada por estudantes do oitavo período, matriculados no estágio INESC. As etapas de elaboração de roteiro textual, com adequação de linguagem e uso da função conativa foram cumpridas, bem como o uso dos recursos lúdicos. De maneira geral, foi percebido, tanto pelos docentes quanto pelos próprios alunos, a potência e o alcance do material utilizando as ferramentas digitais, que além de transformarem e enriquecerem a formação acadêmica, preparando os estudantes para uma nova realidade póspandemia, também são ferramentas perenes de transformação social, podendo ser utilizadas em diversos contextos e locais, ampliando o acesso da população às medidas educativas em saúde bucal Descritores: Educação em Saúde. Saúde Pública. Estratégias de Saúde Digital. Saúde Bucal.
\end{abstract}

\section{INTRODUÇÃO}

Declarada como uma pandemia em março de 2020, a doença causada pelo novo coronavírus (COVID-19) acabou mudando drasticamente os serviços de saúde, que por medida de segurança impediram a atuação de estagiários na rede de saúde, visando à redução da aglomeração de pessoas, o que tornou obrigatória a discussão de adaptação de métodos e ferramentas de trabalho inovadoras para dar continuidade às atividades educativas, diminuindo a probabilidade de infecção pelo vírus SARS-COV-2 ${ }^{1-3}$. As instituições de ensino superior, inclusive no âmbito dos cursos de Odontologia, especialmente pela presença de aerossóis em sua prática cotidiana e o contato próximo entre 
estudantes/professores e pacientes, tiveram que suspender suas atividades clínicas, teóricas e práticas por meio da portaria 343 do 17 de março de $2020^{21}$, realidade que não foi diferente no Centro Universitário de Patos de Minas (UNIPAM). Dentre as diversas atividades suspensas, uma delas foi o componente curricular Estágio Integração, Ensino, Serviço e Comunidade (INESC).

Considerada, atualmente, uma das maiores ferramentas digitais, a internet permite usar a interatividade no ambiente lúdico para ensino, formação de identidade pessoal e ainda uma comunicação sincronizada, facilitando as relações de comunicação e atividades, principalmente no período de crise sanitária e de relações humanas e educacionais que foi disparada pela pandemia e seus desdobramentos como o isolamento sociall ${ }^{4-6}$.

A educação em saúde é definida como um processo de construção de conhecimentos, que aumenta a autonomia das pessoas no seu cuidado ${ }^{7}$ Nesse processo, o uso das ferramentas digitais como coadjuvantes ao ensino e técnicas educativas tradicionais, permite inúmeras vantagens voltadas à facilidade e comodidade dos processos de aprendizagem e aquisição e informações em saúde, viabilizando uma maior flexibilização de horários e concedendo acesso a essas informações em qualquer lugar ${ }^{6,8}$.

Ferramentas que ganharam grande repercussão foram as tecnologias digitais da informação e comunicação (TDIC), as quais permitem inúmeras inovações, além de desenvolverem novas maneiras de aprender ${ }^{6,9}$. Recursos audiovisuais, como vídeos educativos, significam uma sofisticação na relação ensinoaprendizagem, já que por meio deles consegue-se captar a atenção do público, bem como despertar sua curiosidade em relação às temáticas abordadas, explorando a habilidade visual e a capacidade de processar informações ${ }^{10}$.

Diante deste panorama, é valorizada a aprendizagem colaborativa, a realização de projetos em grupo, assim como o estabelecimento de redes sociais de trabalho. $\mathrm{O}$ estudante atual identifica o seu colega como uma importante fonte de aprendizagem. Desta forma, as redes sociais virtuais permitem tornar as ferramentas de interação importantes dentro e fora da sala de aula, além de uma comunicação facilitada e integral, esta que se trata da habilidade de observar e identificar necessidades reais e agir para alcançar o objetivo estabelecido para aquela comunicação específica com o paciente $e^{11,12}$.

Neste contexto, o objetivo deste artigo é relatar a experiência da aplicação dos conceitos de educação em saúde no ensino remoto por meio da confecção de vídeos de criação compartilhada por estudantes do oitavo período, matriculados no estágio INESC do Curso de Odontologia do UNIPAM.

\section{RELATO DE EXPERIÊNCIA}

A Organização Mundial da Saúde (OMS) declarou, em 30 de janeiro de 2020, que o surto da COVID-19 constitui uma emergência de Saúde Pública de importância internacional ${ }^{13}$. Em março de 2020 foi elevado ao estado de pandemia pelo alto potencial de transmissibilidade do vírus SARS-CoV-2, levando governos e instituições, em todo o mundo, a adotar medidas de controle e prevenção para conter o avanço da pandemia, limitando o funcionamento de estabelecimentos e serviços $^{14-18}$

A fim de permitir o contínuo processo de ensino, o Ministro de Estado da Educação, por meio da portaria $\mathrm{n}^{\circ} 343$, de 17 de março de 2020 , autorizou a substituição das aulas presenciais por aulas em meios digitais enquanto durar a situação de pandemia ${ }^{2,19}$. A Associação Brasileira de Ensino Odontológico (ABENO), alinhada com a Association for Dental Education in Europe (AADEE) e a American Dental Education Association (ADEA), apoiou o ensino remoto 
emergencial $^{20}$.

Nesse contexto, iniciaram-se debates sobre alternativas que permitissem dar continuidade ao processo de ensino-aprendizagem no meio acadêmico. A partir dessa discussão, surgiu o regime letivo remoto $(\mathrm{RLR})^{21}$, que utiliza tecnologias digitais para promover a transmissão de informações entre discentes e docentes de maneira síncrona e assíncrona ${ }^{22}$.

Integrando a matriz curricular do Curso de Odontologia do Centro Universitário de Patos de Minas, o estágio INESC proporciona aos alunos, do primeiro ao oitavo período, a experiência de atuarem nas Unidades de Saúde da Família (USF). Dentre as atribuições estão, principalmente, as atividades de educação em saúde e atendimentos clínicos à população local. Todavia, a maioria das atividades teve de ser suspensa de sua forma presencial, conforme recomendações das autoridades sanitárias para conter a propagação do novo coronavírus ${ }^{23}$.

Como alternativa para que o processo ensino-aprendizagem não fosse interrompido e nem a população ficasse sem acesso a ações educativas, foi proposto aos estudantes do oitavo período a elaboração de vídeos para confecção de material educativo em saúde bucal, para diferentes públicos, ciclos de vida e temáticas.

Tais vídeos com conteúdos educativoexplicativos abrangeram diferentes temas pertinentes à saúde bucal, estabelecidos pelo Manual Técnico de Educação em Saúde Bucal $(2007)^{24}$ : técnicas de escovação/higienização; prevenção da cárie dental; diabetes e doença periodontal; prevenção ao tabagismo e câncer de boca.

Por meio da plataforma do Google Meet, de forma síncrona, na turma do oitavo período, foram formados 5 grupos compostos por 11 a 12 alunos, e para cada grupo foi designado um professor orientador para nortear a pesquisa e programação da atividade. Os temas foram distribuídos por meio de sorteio.

Para elaboração de vídeos educativos são preconizadas cinco etapas: análise e planejamento; modelagem; implementação; avaliação e manutenção, e distribuição. Seguindo essa sequência, na etapa de análise e planejamento, durante a reunião do grupo, foi proposto o planejamento de um script com seleção adequada de imagens para o vídeo, produção de um texto acessível e uma linguagem técnicocientífica adaptada e voltada para o público ${ }^{25,26}$.

Após a confecção do script pelos alunos, na etapa de modelagem dos textos, os professores orientadores sugeriram alterações que fossem baseadas em evidências científicas. A principal alteração foi em relação à linguagem muito técnica e inacessível ao público-alvo, além de pontuar sobre a capacidade de síntese destacando apenas os pontos relevantes do tema.

No processo de implementação, foram apresentadas alternativas para a confecção dos vídeos: Powtoon $^{\circledR}$, Canva $^{\circledR}$, Videoscribe $^{\circledR} \mathrm{e}$ Powerpoint $^{\circledR}$. Ademais, durante a confecção da atividade, buscou-se outras plataformas que permitissem desenvolver o vídeo de uma forma mais dinâmica e fácil: Animaker ${ }^{\circledR}$, Rawshorts ${ }^{\circledR} \mathrm{e}$ Moviemaker $^{\circledR}$. Como uma das regras para confecção do vídeo, foi orientado o tempo limite de 5 minutos, no qual fosse apresentado o vídeo na íntegra abordando a temática definida.

No processo de escolha da plataforma foram considerados alguns pré-requisitos para definir qual integrante do grupo seria responsável pela confecção do vídeo: maior familiaridade com a tecnologia, interesse em aprender e participar da criação, plataforma de fácil manuseio e aplicativos acessíveis. Foi designado a cada membro ajudar na elaboração do vídeo seja na etapa planejamento, seja na modelagem, seja no processo de implementação. Dessa forma, foi necessário que dois alunos fizessem as gravações do texto do script para que fossem colocadas 
como vozes no fundo do vídeo, simulando diálogos, frases e mensagens.

Para dar andamento ao processo de elaboração do vídeo, foram necessárias pesquisas acerca do uso da plataforma escolhida pelo grupo, bem como suas funcionalidades e aplicabilidades. Integrantes de diferentes grupos relataram dificuldades, em reuniões síncronas de acompanhamento e avaliação junto ao tutor, quanto à melhor distribuição das falas nos vídeos, sincronização das falas do texto com as vozes dos alunos, conversão dos áudios para o formato que a plataforma requeria, e dificuldade em selecionar imagens e fontes que fossem atrativas para o público. Dessa forma, após o debate de ideias, as dúvidas puderam ser solucionadas e a atividade continuada.

No dia de entrega foi apresentado o conteúdo final para toda a turma, por meio do Google Meet, sob supervisão dos professores orientadores. Foi necessário enviar os vídeos por e-mail, para que fosse feita a avaliação e divulgação nas plataformas digitais, Instagram ${ }^{\circledR} \mathrm{e}$ Youtube $^{\circledR}$, nos quais foram criados respectivamente uma página denominada "INESC Odonto" UNIPAM e um canal chamado "INESC Odonto" para a divulgação em larga escala. Neste mesmo encontro síncrono, utilizando a roda de conversa, ainda que de maneira virtual, os alunos foram questionados sobre sua percepção acerca do uso dos vídeos para ações educativas em saúde, e verbalmente demostraram que acreditavam na potencialidade do uso de tais ferramentas para manutenção das ações de educação em saúde, e em sua abrangência, pois diferente de ações presenciais, nas quais todos precisam estar no mesmo espaço, ao mesmo tempo, o uso de ferramentas digitais permite que as pessoas possam se utilizar da informação a qualquer momento e de maneira contínua, dado sua publicação em mídias sociais. Cópias dos vídeos foram cedidas para a
Secretaria Municipal de Saúde de Patos de Minas, para utilização pelos profissionais das equipes de saúde bucal em atividades educativas, em âmbito da sala de espera, grupos operativos ou mesmo em visitas domiciliares. Essa estratégia se baseia na premissa de que alguns pacientes podem não possuir equipamentos para visualização do vídeo por conta própria, ou ainda serviço de internet, e neste caso podem ter acesso à informação quando da visita à Unidade de Saúde, ou mesmo durante a visita domiciliar.

O último passo da atividade foi de distribuição/divulgação do vídeo nas plataformas do curso. Tais ferramentas digitais são vistas como facilitadoras para o aprendizado, além de serem populares e facilmente acessadas, o que acabou gerando a disseminação da temática e compartilhamento do saber entre diversos alunos ${ }^{11}$. Os estudantes puderam ter a experiência da criação compartilhada de materiais didáticos, por meio de ferramentas que lhe são conhecidas, já que em sua maioria estão familiarizados com tecnologias digitais e possuem facilidade na utilização da internet e de todas as suas facilidades e recursos.

\section{CONSIDERAÇÕES FINAIS}

De maneira geral, foi percebido, tanto pelos docentes quanto pelos próprios alunos, a potência e o alcance do material utilizando as ferramentas digitais, especialmente as audiovisuais como os vídeos para serem divulgados via Instagram ${ }^{\circledR} \mathrm{e}$ Youtube $^{\circledR}$, que transformam e enriquecem a formação acadêmica preparando os estudantes para a realidade pós-pandemia, e preparam para o mercado de trabalho. Além disso os vídeos ainda se constituem como possíveis ferramentas perenes de transformação social, podendo ser utilizadas em diversos contextos e locais, amplificando o acesso da população às medidas educativas. Foram adotadas estratégias de distribuição dos vídeos para que a divulgação da informação fosse 
a mais democrática possível, mesmo para pacientes sem acesso a equipamentos eletrônicos como smartphones ou mesmo à internet.

\section{ABSTRACT \\ Videos as tools for the education in oral health in the teaching-service integration at the University Center of Patos de Minas during the pandemic}

In the graduate course in Dentistry at the University Center of Patos de Minas, several inperson activities of the Teaching, Service, and Community Integration (INESC) internship had to be suspended due to the COVID-19 pandemic, as recommended by health authorities. This article aims to report the experience of applying the concepts of health education in remote education through the production of videos shared by eighth-period students enrolled in this internship. The steps for preparing a script, with language adaptation, and the use of the conative function were completed, as well as the use of playful resources. In general, teachers and students have perceived the power and reach of digital tools, which, in addition to transforming and enriching the academic training and preparing students for a new post-pandemic reality, are also perennial tools of social transformation, that can be used in different contexts and places, expanding the access of the population to educational measures in oral health.

Descriptors: Health Education. Public Health. Digital Health Strategies. Oral Health.

\section{REFERÊNCIAS}

1. Faria MHD, Pereira FJP, Silva ML, Pessoa DM, Soares SCM. Relato de alunos de odontologia no enfrentamento à covid-19. Cadernos ESP. 2020; 14(1):118-22.

2. Fernandez MS, Silva NRJ, Viana VS, Oliveira CCC. Doença por coronavírus 2019: desafios emergentes e o ensino odontológico brasileiro. Rev ABENO. 2020; 20(2): 2-15.

3. Giudice, RL. The severe acute respiratory syndrome coronavirus-2 (sars cov-2) in dentistry. Management of biological risk in dental practice. Int $\mathrm{J}$ Environ Res Public Health. 2020;17(9):3067.

4. Zimmer R, Almeida Neto $\mathrm{H}$, Reston EG, Klein Júnior AC. O papel das mídias sociais na construção do conhecimento em Odontologia. Stomatos. 2018; 24(47):1-5.

5. Santana VV, Santos PR, Leal AKTBN, Silva DBS, Pereira EV, Silveira LNS, et al. A importância do uso da internet sob o viés da promoção interativa na educação em tempos de pandemia. Braz J Develop. 2020; 6(10):78866-76.

6. Xavier TB, Barbosa GM, Meira CLS, Conte Neto N, Pontes HAR. Utilização de recursos web na educação em Odontologia durante pandemia COVID-19. Braz J Health Rev. 2020; 3(3):4989-5000.

7. Ministério da Saúde. Secretaria-Executiva. Secretaria de Gestão do Trabalho e da Educação na Saúde. Glossário temático: gestão do trabalho e da educação na saúde. Ministério da Saúde: Brasília. 2012, 44(2) (Série A. Normas e Manuais Técnicos).

8. Faleiro FRG, Salvago BM. Educação a distância nos cursos de graduação em odontologia no Brasil. Rev Bras Aprendiz Aberta Dist. 2018; 17(1):1-28.

9. Gusso HL, Gonçalves VM. Ensino superior em tempos de pandemia: diretrizes à gestão universitária. Educ Soc. 2020; 41: 1-26.

10. Rodrigues Junior JC, Rebouças CBA, Castro RCMB, Oliveira PMP, Almeida PC, Pagliuca LMF. Construção de vídeo educativo para a promoção da saúde ocular em escolares. Texto Contexto Enferm, 2017, 26(2):e06760015.

11. Gibran A. Comunicação integral: o que é isso? [Acesso em 24 out. 2021]. Disponível em: https://andregibran.com/o-que-e-comunicacaointegral/.

12. Souza FB, Lopes MGQ, Lima Filho RM. 
Redes sociais na aprendizagem em odontologia: opinião dos estudantes de uma universidade brasileira. Rev Cuba Estomatol. 2017; 54(2):1-11.

13. World Health Organization - WHO. Novelcoronavirus-2019. 2019. [Acesso em 13 abr. 2021]. Disponível em: https://www.who.int/e megencies/diseases/novel-coronavirus-2019.

14. Ge H, Wang X, Yuan X, Gong X, Wang C, Deng $\mathrm{T}$, et al. The epidemiology and clinical information about Covid-19. Eur J Clin Microbiol Infect Dis. 2020; 39(6):1011-19

15. Rothan HA, Byrareddy SN. The epidemiology and pathogenesis of coronavirus disease (Covid-19) outbreak. J Autoimmun. 2020; 109:102433.

16. Sohrabi C, Alsafi Z, O’Neill N, Khan M, Kerwan A, Al-Jabir A, et al. World Health Organization declares global emergency: a review of the 2019 novel coronavirus (Covid19). Int J Surg. 2020; 76:71-6.

17. Garcia LP, Duarte E. Intervenções não farmacológicas para o enfrentamento à epidemia da Covid-19 no Brasil. Epidemiol Serv Saúde. 2020;29(2):e2020222.

18. Aquino EML, Lima RTRS. Medidas de distanciamento social no controle da pandemia de Covid-19: potenciais impactos e desafios no Brasil. Ciênc Saúde Colet. 2020; 25(1):2423-46.

19. Gomes VTS, Rodrigues RO, Gomes RNS, Gomes MS, Viana LVM, Silva FS. The covid19 pandemic: repercussions of remote education on medical training. Rev Bras Educ Méd. 2020; 44(4):e114.

20. Associação Brasileira de Ensino Odontológico. Posicionamento da ABENO sobre a substituição das aulas presenciais por aulas por meios digitais nos cursos de Odontologia, enquanto durar a situação de pandemia COVID-19. 2020. [Acesso em 23 jun. 2021]. Disponível em: https://abeno.org. br/abeno-files/downloads/posicionamento-ab eno-portaria-mec-544.pdf.

21. Vercelli LCA. Aulas remotas em tempos de Covid-19: a percepção de discentes de um programa de mestrado profissional em educação. @mbienteeducação 2020; 13(2):47-60.

22. Alves L. Educação remota: entre a ilusão e a realidade. Educação. 2020; 8(3):348-65.

23. Brasil. Portaria MEC no 343 , de 17 de março de 2020. [Acesso em 23 jun. 2021]. Disponível em: https://abmes.org.br/legisla coes/detalhe/3017/portaria-mec-n-343.

24. SESC. DN. DPD Manual técnico de educação em saúde bucal / Claudia Márcia Santos Barros, coordenador. - Rio de Janeiro : SESC, Departamento Nacional, 2007.

25. Falkembach GAM. Concepção e desenvolvimento de material educativo digital. Renote. 2005; 3(1).

26. Razera APR, Buetto LS, Lenza NFB, SonobeHM. Vídeo educativo: estratégia de ensino-aprendizagem para pacientes em tratamento quimioterápico. Ciênc Cuid Saúde 2014; 13(1):173-8.

\section{Correspondência para:}

Thiago de Amorim Carvalho e-mail: thiagocarvalho@ unipam.edu.br

Centro Clínico Odontológico

Centro Universitário de Patos de Minas

Av. Marabá, 831 Alto Caiçaras

38703-236 Patos de Minas/MG 